Journal of Applied Fluid Mechanics, Vol. 3, No. 2, pp. 23-32, 2010.

Available online at www.jafmonline.net, ISSN 1735-3572, EISSN 1735-3645.

DOI: $10.36884 / \mathrm{jafm} .3 .02 .11885$

\title{
Characterization of Flow through the Intake Valve of a Single Cylinder Engine Using Particle Image Velocimetry
}

\author{
B.M. Krishna* and J.M. Mallikarjuna \\ Internal Combustion Engine Laboratory, Department of Mechanical Engineering \\ Indian Institute of Technology Madras, Chennai - 600 036, India \\ Email: " murali2kindia@gmail.com
}

(Received September 6, 2008; accepted June 22, 2009)

\begin{abstract}
This paper deals with the investigations of the in-cylinder flow pattern around the intake valve of a single-cylinder internal combustion engine using Particle Image Velocimetry (PIV) at different intake air flow rates. The intake air flow rates are corresponding to the three engine speeds of 1000, 2000 and $3000 \mathrm{rev} / \mathrm{min}$., at all the static intake valve opening conditions. In this study, in-cylinder flow structure is characterized by the tumble ratio and maximum turbulent kinetic energy of the flow fields. In addition, at two specified lines of the combustion chamber, the radial and axial velocity profiles have been plotted. From the results, it is found that the overall airflow direction at the exit of the intake valve does not change significantly with the air flow rate and intake valve opening conditions. The tumble ratio increases with increase in intake valve opening and not much affected by the change in the air flow rates. It is also found that, the variations of the velocity profiles at the two specified lines are smooth at full intake valve opening irrespective of the air flow rate. Also, their magnitudes increase with increase in the intake valve openings at all the air flow rates. The in-cylinder flow analysis carried out in this study may be useful for optimizing the intake valve opening of an internal combustion engine with respect to it's speed.
\end{abstract}

Keywords: Inlet valve, valve opening, engine speed, PIV, raw image, velocity field

\section{INTRODUCTION}

The main objective of any Internal Combustion (IC) engine is to obtain good performance and meet the stringent emission norms. In order to achieve this, combustion process is very vital in the IC engines. However, the combustion in turn is mainly affected by the in-cylinder flow structure. In an IC engine, the air enters the combustion chamber through the intake port with high velocity during the intake stroke. Thus high kinetic energy of the fluid flow during intake stroke, later results in high turbulence.

In-cylinder fluid flows govern the flame propagation rate in the spark ignition (SI) engines and control the air-fuel mixing in the diesel engines (Heywood, 1988). In addition, it controls the heat transfer to the cylinder walls, the combustion process and finally the formation of emissions. In IC engines, induction of air is of greater importance than that of fuel even though air and fuel are vital substances in the combustion process. Study of complex in-cylinder fluid flow structures in an IC engine requires sophisticated instrumentation.
Previous investigations on in-cylinder flow studies in the IC engines have reported that the large-scale fluid flows (swirl and tumble) generated during the intake stroke were later dissipated into turbulence during the compression stroke (Li et al., 2002; Yasar et al., 2006; Valentino et al., 1993; Lee et al., 1993 and Stansfield et al., 2007). High swirl and tumble flows are known to produce very large air velocities and high dissipation rates (Reuss et al., 1995). Abdullah et al. (2008) compared the in-cylinder flow structures obtained by Particle Image Velocimetry (PIV) with that of Computational Fluid Dynamics (CFD), and also analyzed the combustion process and emission characteristics in a compressed natural gas direct injection engine. Their results showed that retarding injection and ignition timings could reduce $\mathrm{CO}$ and NOx levels significantly with good engine performance.

Engine in-cylinder flow studies using Particle Tracking Velocimetry (PTV) reported that the combustion control could be achieved through the turbulence in a premixed lean burn engine and by effective air-fuel 
mixing in a gasoline direct injection (GDI) engine (Kuwahara and Ando, 2000). Laser Doppler Anemometry (LDA) investigations on swirling flows through an axi-symmetric port and poppet valve reported that the swirl alters the flow structure below the intake valve significantly. Also, both the mean tangential and axial velocities increase during the intake valve opening period; reach the peak value at the time of intake valve closure (Nadarajah et al., 1998; Zhu et al., 1990 and Auriemma et al., 2001).

Previously many experimental and numerical investigations by different techniques were addressed the issues of in-cylinder flows in the IC engines. However, still there is a good scope to study the incylinder flows under different conditions. Therefore, the present study deals with the in-cylinder flows using PIV technique under different air flow rates corresponding to the different engine speeds (from now onwards stated as equivalent engine speed) with different static intake valve opening conditions.

\section{Experimental Procedure}

The PIV system and photographic view of the experimental setup used in this study are shown in Figs. 1 and 2 respectively. In this study, in-cylinder flow investigations were carried out at the exit of the intake valve of a single-cylinder IC engine at different air flow rates corresponding to the equivalent engine speeds of 1000,2000 and $3000 \mathrm{rev} / \mathrm{min}$., with 50, 75 and $100 \%$ static intake valve opening conditions using PIV technique. The intake valve of axi-symmetric type with a maximum lift of $7.6 \mathrm{~mm}$ was used. An adjustable valve opening arrangement (Fig.2) was developed and used. To facilitate the PIV measurements, the cylinder was made of transparent material (Plexiglass) with inside diameter of $87.5 \mathrm{~mm}$ and length of $110 \mathrm{~mm}$. It was attached to the actual engine cylinder head. The intake manifold of the test unit was connected to a steady flow test rig, which consists of an air-blower and a settling chamber followed by a diffuser and a contraction. An orifice meter was used for the measurement of air flow rate.

The PIV system consists of a double pulsed ND-YAG laser of $200 \mathrm{~mJ} /$ pulse energy at $532 \mathrm{~nm}$ wave length, a charge coupled device (CCD) camera of resolution $2048 \times 2048$ pixels with 14 frames per second, laser and camera controllers, and a data acquisition system with a software. Triggering of the laser and camera was controlled by a LaVision DAVIS (data acquisition and visualization) software. In this work, Di-Ethyl-HexylSebacat $\left(\mathrm{C}_{26} \mathrm{H}_{50} \mathrm{O}_{4}\right)$ was used as a seeding fluid of particle size of one micrometer diameter generated by a particle generator and was mixed with the supplied air in the intake manifold at an appropriate place. The laser sheet was allowed to pass through the transparent cylinder on a vertical plane passing through the axis of the intake valve. Figure 3 shows a typical raw image of the Field of View (FOV) at full intake valve opening condition. The zone near the exit of the intake valve was considered as the Region of Interest (ROI) to study the flow characteristics as shown by the dotted lines in Fig.3. At every test condition, 150 image pairs were recorded. The required air flow rate at various equivalent engine speeds were calculated by assuming an engine volumetric efficiency of $90 \%$. The time interval $(\Delta t)$ between the two images of an image pair was estimated based on the air flow velocity, FOV, pixel shift, image size and camera resolution (LaVision, 2006).

\section{Results AND Discussion}

Post processing and analysis of the data was carried out for 150 image pairs using DAVIS software. During the post processing, a multi-pass, cross-correlation technique with a size of $32 \times 32$ pixel interrogation window and 50\% overlap was considered (Raffel, 1998 and LaVision, 2006). From the instantaneous velocity vector fields, the average velocity vector fields with streamline patterns, tumble ratio (TR), maximum turbulent kinetic energy (TKE), and radial and axial velocity components were evaluated, plotted and discussed in the following sections.

\subsection{In-cylinder Flow Pattern}

Figures 4 to 6 show velocity vector plots for three values of intake valve openings and three equivalent engine speeds. From, Fig. 4, it is observed that the overall direction of velocity vectors was almost identical for all the three equivalent engine speeds at full intake valve opening. Similar observations were made with other intake valve openings also. However, there was a noticeable reduction in tumble ratio at half intake valve opening compared to other openings (about $62.5 \%$ reduction). Hence, the velocity vector plots for the other intake valve openings are shown at an equivalent engine speed of $1000 \mathrm{rev} / \mathrm{min}$ (Figs.5 and 6).

Also, from these figures, it is observed that there is a formation of an air jet at the exit of the intake valve. Later, this air jet interacts and mixes with the surrounding bulk air and diffuses into it (Murali Krishna et al., 2008). However, the magnitude of air velocity increases with increase in equivalent engine speed and intake valve opening.

Figure 4 shows the ensemble average velocity vectors under full intake valve opening condition for different intake air flow rates (equivalent engine speeds). From Fig.4, it is seen that a clock wise $(\mathrm{CW})$ vortex is forming below the intake valve as expected (Heywood, 1988). Also, it shows the presence of an orderly flow pattern near the bottom right corner of the intake valve with a single $\mathrm{CW}$ vortex. This is due to the impingement of the high velocity air jet emerging from the intake valve port onto the right side cylinder wall. The emerging jet of air is deflected downwards by the right side cylinder wall resulting in $\mathrm{CW}$ vortex formation. This $\mathrm{CW}$ tumble vortex is shifting towards right side with increase in the equivalent engine speed as shown in Fig.4. Further, it is leading to the formation of number of small and large-scale vortices at high equivalent engine speeds.

Figure 5 shows the in-cylinder flow structure under $3 / 4^{\text {th }}(75 \%)$ intake valve opening condition for an equivalent engine speed of $1000 \mathrm{rev} / \mathrm{min}$. From Fig. 5, 
it is observed that there is no clear vortex formation as in the case of full intake valve opening condition. Also from Fig. 5, it is observed that the flow is more random in nature. However, it looks like there are many small sized vortices in the flow field. This trend is observed for all the air flow rates considered in this study at $75 \%$ intake valve opening condition. This may be due to the fact that, at $75 \%$ intake valve opening condition, intake valve opening is comparatively less restricting the flow of air into the cylinder. Also, the air entering the cylinder is tending to move horizontally and striking the right cylinder wall rather moving downwards. It is observed by the in-cylinder flow studies that, another reason for the formation of the $\mathrm{CW}$ vortex below intake valve is the due the low pressure zone just below the intake valve. When more amount of air flow towards right side; it may leads to the formation of low pressure region below the intake valve making air to rush from the other regions causing more randomness in the incylinder air flow pattern.

Figure 6 shows the in-cylinder flow structure at half intake vale opening $(50 \%)$ condition for an equivalent engine speed of $1000 \mathrm{rev} / \mathrm{min}$. In this case also, flow structure is more random in nature as in the case of $75 \%$ intake valve opening condition. This may be again due to small opening of the intake valve causing the air to move much horizontal almost parallel to the cylinder head. Also, it may be due to the similar reasons as stated above ( $75 \%$ opening case).

In this study, characterization of the in-cylinder tumble flows has been done by the tumble ratio (TR) based on an equation proposed by Huang et al. (2005). Figure 7 shows the variation of the tumble ratio with the intake valve opening at different air flow rates. From Fig.7, it is observed that TR is higher at higher intake valve opening conditions compared to lower ones irrespective of the air flow rates. This may be because of the increased air flow rate and velocity through the intake port at higher intake valve opening conditions. In addition, when the intake valve opening is small, there can be loss of pressure and velocity due to fluid friction with the metal walls. These losses may reduce at higher intake valve openings. At higher intake valve opening, mass flow rate of air increases which also increases the momentum of the flow. This is responsible for accelerating the flow into the cylinder. Along with high air velocity, the low pressure zone below the intake valve may also cause the formation of in-cylinder tumble vortex.

The reduction in tumble ratio with rise in engine speed is evident from Fig. 7. It may be due to the reduction in the radius of single large vortex. The structure and pattern of the in cylinder flow field, however, did not change much with engine speed, but the center of single large vortex moves towards right causing its radius to reduce, owing to higher jet velocity. This may cause a slight reduction in tumble ratio with increase in engine speed. Almost same trend is observed for tumble ratio at other air flow rates considered. Therefore, it seems that tumble ratio is the strong function of the intake valve lift than the air flow rate.
Generally, for SI engines, it is suggested to have a strong tumble motion during suction stroke with high kinetic energy which will be later dissipated as turbulence during the compression stroke at the point of spark (Khalighi, 1991). High turbulence is very much helpful in increasing the flame speed in stratified and direct injection SI engines.

\subsection{Maximum Turbulent Kinetic Energy}

In an IC engine, turbulence controls the mixing (in direct injection engines), heat transfer and flame speed (Kuwahara, 2000). It is quantified by the maximum turbulent kinetic energy (TKE) of the in-cylinder flows. Here, TKE has been calculated for various intake valve openings at various air flow rates from the root mean square (rms) velocity of the average velocity vector fields at the mid-plane of intake valve based on the equations proposed by Reuss et al. (1995).

Figure 8 shows the plot of maximum TKE at various equivalent engine speeds at different intake valve opening conditions. From Fig. 8, it is observed that the maximum TKE is higher at higher equivalent engine speed (3000 rev/min) than at lower equivalent engine speeds irrespective of the intake valve opening. Similarly, irrespective of the equivalent engine speed, magnitudes of maximum TKE are increasing with increase in intake valve opening. It may be due to high air velocity at the intake valve exit at higher equivalent engine speeds and intake valve openings.

\subsection{Velocity Profiles}

Here, radial and axial velocity components have been estimated from the ensemble average velocity vectors at a distance of $3 \mathrm{~mm}$ away from the intake valve tip on one horizontal and one vertical line (Fig.3). These velocity profiles are shown in Figs.9 to 11. These are useful for the estimation of velocity gradient properties like vorticity and strain rates which influence the fundamental characteristics of turbulence.

From Figs. 9 to 11, it is observed that both the axial and radial velocity profiles are smooth at higher intake valve openings irrespective of the equivalent engine speeds. However, the magnitudes of them are increasing with increase in intake valve openings and equivalent engine speeds. This may be due to the higher air flow rates at higher equivalent engine speeds; since the absolute velocities are higher at higher equivalent engine speeds at a given intake valve opening. At lower intake valve opening (at 50 and $75 \%$ ), the axial and radial velocity profiles are not so smooth. This may be due to randomness in the in-cylinder flows as explained above. It is known that when fluid passes over a bluff body, recirculation zones will be formed around it. In addition, at lower intake valve opening, the flow velocities are less due to the fluid friction caused by fluid and metal wall interaction. Since the inlet valve and ports are the largest flow restrictions in the intake system which results in the large pressure drop across the intake valve. However, these problems are less at higher intake valve opening; may be resulting in smooth profiles of radial and axial velocities. 
From the above discussion, it follows that in order to have good tumble flow during intake stroke; the engine should be operated with a large intake valve opening irrespective of the engine speed. Since the investigations carried out in this study are under static intake valve opening conditions, in order to verify the validity of the above experimental findings for actual operating conditions, it is suggested that the in-cylinder flow investigations under real engine conditions are to be carried out for a set of typical operating conditions to optimize intake valve opening.

\section{Conclusions}

In this study, PIV tests were performed to study the incylinder tumble flow structures for various intake valve openings at different air flow rates. Based on the results, the following conclusions are drawn:

- The overall air flow pattern at the exit of the intake valve is identical for all the equivalent engine speeds at full intake valve opening.

- The radius of the single large vortex below intake valve increases with increase in intake valve opening.

- Tumble ratio is higher at higher intake valve opening at the given equivalent engine speed.

- Tumble ratio is not much affected by the air flow rates (equivalent engine speed) at the given intake valve opening.

- The maximum TKE of the flow field increases with increase in equivalent engine and intake valve opening.

- The radial and axial velocities near intake valve exit are increasing with increase in equivalent engine speeds and intake valve opening.

On whole, the flow analysis carried in this work may be useful in optimizing the intake valve opening.

\section{ACKNOWLEDGEMENTS}

The authors are thankful to the staff members of IC Engines Lab, IIT Madras, Mr. K. Nagarajan, Mr. M. K. Subramanian, Mr. M. Michael John Bose and Mr. S. Babu, who helped to carry out the experiments.

\section{REFERENCES}

Abdullah, S., W.H. Kurniawan and A. Shamsudeen (2008). Numerical Analysis of the Combustion Process in a Compressed Natural Gas Direct Injection Engine. Journal of Applied Fluid Mechanics 1(2), 65-86.

Auriemma, M.F., E. Corcione, U.Di Martino and G. Valentino (2001). Analysis of the intake flow in a diesel engine head using dynamic steady flow conditions, SAE Paper No. 2001-01-1307.

LaVision (2006). DAVIS Software Manual, M/s LaVision GMBH, Germany.
Heywood, J.B. (1988). Internal Combustion Engine Fundamentals, McGraw Hill International Editions.

Huang, R.F., C.W. Huang, S.B. Chang, H.S. Yang, T.W. Lin and W.Y. Hsu (2005). Topological flow evolutions in cylinder of a motored engine during intake and compression stroke. Journal of Fluids and Structures 20, 105-127.

Kuwahara, K. and H. Ando (2000). Diagnostics of incylinder flow, mixing and combustion in gasoline engines. Journal of Measurement Science and Technology 11, R95-R111.

Khalighi, B. (1991). Study of the intake tumble motion by flow visualization and particle tracking velocimetry. Journal of Experiments in Fluids 10, 230-236.

Lee, J. and P.V. Farrell (1993). Intake valve flow measurements of an IC Engine using Particle Image Velocimetry, SAE Paper No. 930480.

Li, Y., H. Zhao, Z. Peng and N. Ladommatos (2002). Particle image velocimetry measurement of incylinder flow in internal combustion enginesexperiment and flow structure analysis, Proceedings of the Institution of Mechanical Engineers (IMechE) 216, Part D, D08701.

Murali Krishna, B. and J.M. Mallikarjuna (2008, March). Intake valve flow analysis in a direct injection diesel engine using particle image velocimetry. Proceedings of the $2^{\text {nd }}$ International Conference on Recent advances in experimental fluid mechanics (RAEFM-2008), Vizayawada, India.

Murali Krishna, B. and J.M. Mallikarjuna (2008, March). Optical diagnosis of flow through the intake valve in a direct injection diesel engine. Proceedings of the $15^{\text {Th }}$ ISME International Conference on new horizons of Mechanical Engineering (ISME-2008), Bhopal, India.

Murali Krishna, B. and J.M. Mallikarjuna (2008, July). Effect of engine speed on intake valve flow characteristics of a diesel engine- An analysis using Particle Image Velocimetry. Proceedings of the First International Conference on Emerging Trends in Engineering and Technology (ICETET2008), No. 978-0-7695-3267-7/08 IEEE, Nagpur, India.

Murali Krishna, B., A. Bijucherian and J.M. Mallikarjuna (2008, October). Effect of intake manifold inclination on intake valve flow characteristics of a single cylinder engine using Particle Image Velocimetry. Proceedings of world academy of science, engineering and technology (PWASET 4, ISSN 2070-3740). The $5^{\text {th }}$ International Conference on Emerging Trends in Engineering and Technology (ICETET2008), Venice, Italy. 
B.M. Krishna and J.M. Mallikarjuna / JAFM, Vol. 3, No. 2, pp. 23-32, 2010.

Nadarajah, S., S. Balabani, M.J. Tindal and M. Yianneskis (1998). The effect of swirl on the annular flow past an axisymmetric poppet valve. Proceedings of the Institution of Mechanical Engineers (IMechE) 212, Part C, C04597.

Raffel, M., C. Willet and J. Kompenhans (1998). Particle Image Velocimetry - A Practical Guide, Springer-Verlag Berlin Heidelberg, Germany.

Reuss, L.D., T. Kuo, B. Khalighi, D. Haworth, and M. Rosalik (1995). Particle Image Velocimetry measurements in a high-swirl engine used for evaluation of computational fluid dynamics Calculations, SAE Paper No. 952381.

Stansfield, P., G. Wigley, T. Justham, J. Catto and G. Pitcher (2007). PIV analysis of in-cylinder flow structures over a range of realistic engine speeds, Journal of Experiments in Fluids 43, 135-146.

Valentino, G., D. Kaufman and P. Farrell (1993), Intake valve flow measurements using PIV, SAE Paper No. 932700.

Yasar, A., B. Sahin, H. Akilli, and K. Aydin (2006). Effect of inlet port on the flow in the cylinder of an internal combustion engine. Proceedings of the Institution of Mechanical Engineers 220, Part C, Journal of Mechanical Engineering Science (IMechE), C16904.

Zhu, Y., D. Evers, G. Huigen and G. Hommersom (1990). LDA measurements of steady and unsteady flow through the induction system of a heavy duty diesel engine. SAE Paper No. 901576. 
B.M. Krishna and J.M. Mallikarjuna / JAFM, Vol. 3, No. 2, pp. 23-32, 2010.

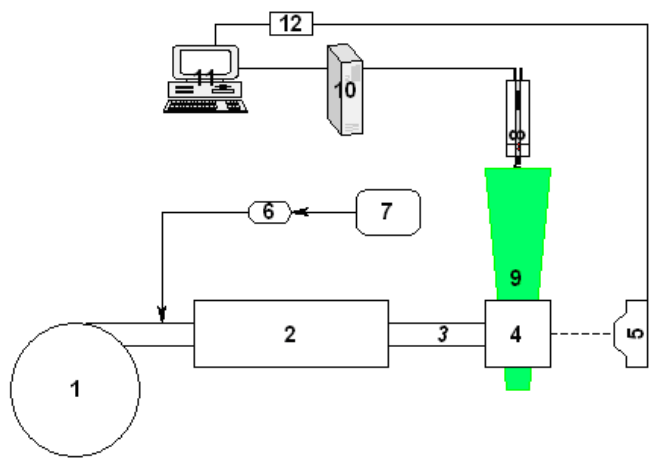

1. Blower, 2. Settling chamber, 3. Connecting pipe 4. Engine, 5. Camera, 6. Seeder,7. Compressor, 8. Nd -YAG Laser, 9. Laser sheet, 10. Laser controller, 11. Acquisition system, 12. Camera controller

Fig. 1. Schematic diagram of the experimental setup

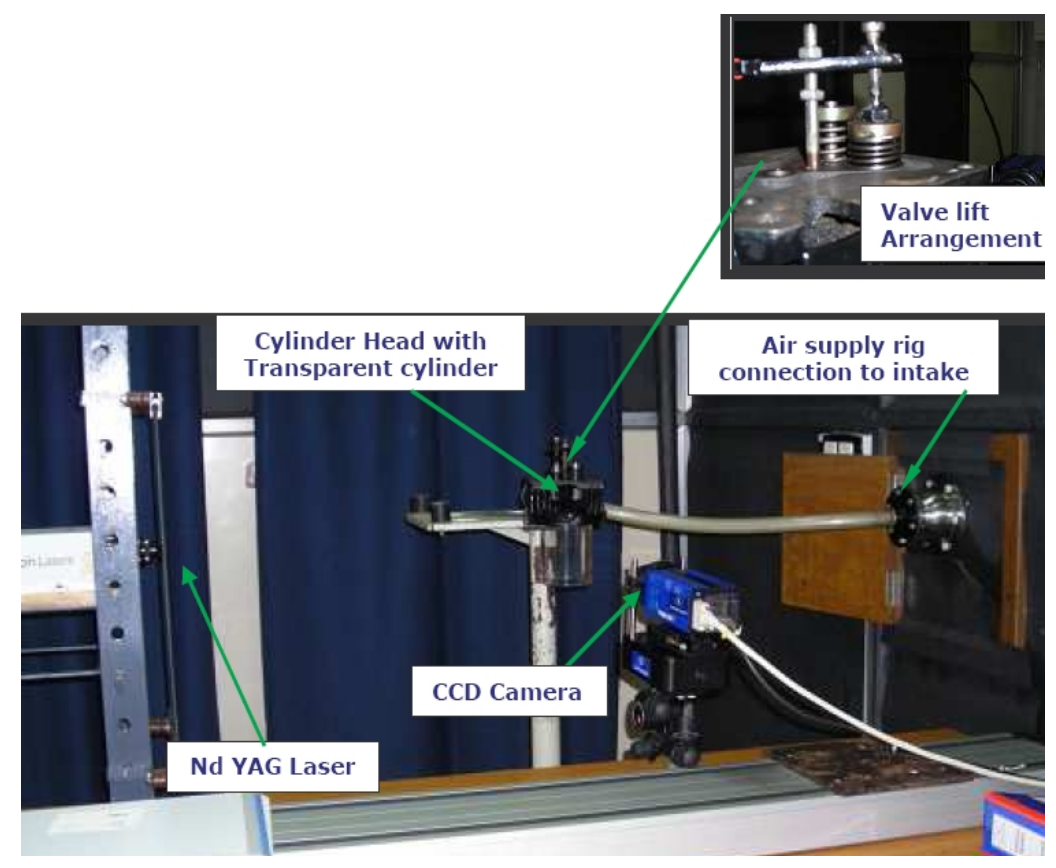

Fig. 2. Photographic view of the experimental setup

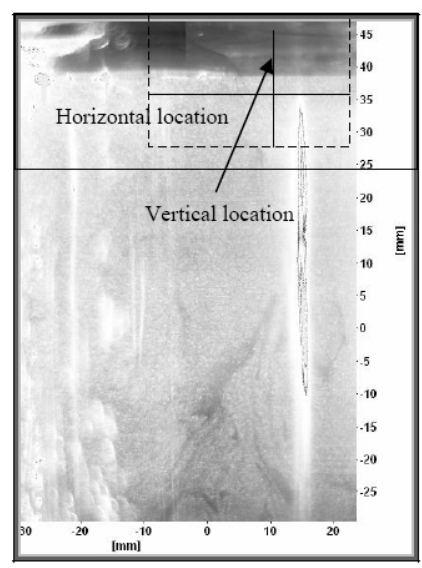

Fig. 3. Typical raw image at full intake valve opening 
B.M. Krishna and J.M. Mallikarjuna / JAFM, Vol. 3, No. 2, pp. 23-32, 2010.
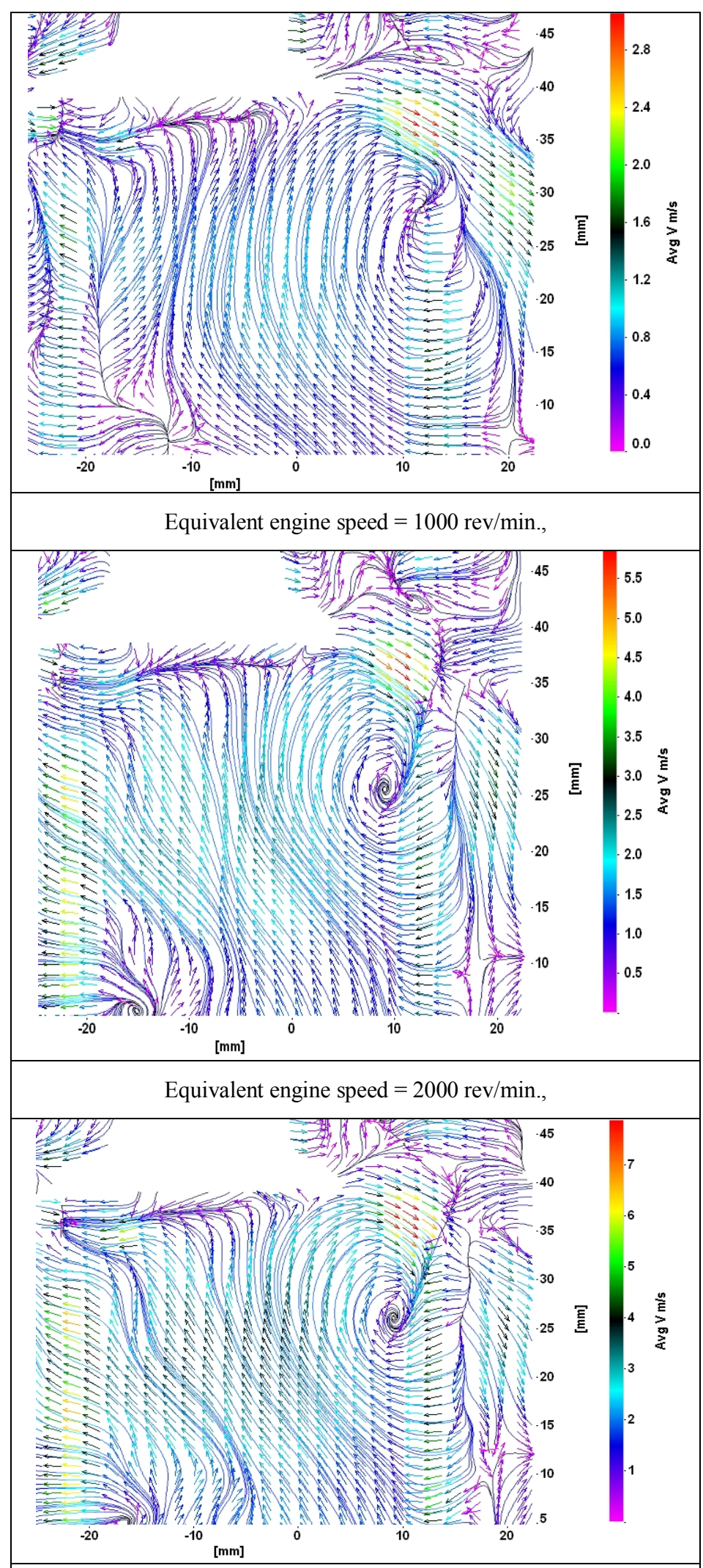

Equivalent engine speed $=3000 \mathrm{rev} / \mathrm{min}$.,

Fig. 4. Ensemble average velocity vector fields at full intake valve opening 
B.M. Krishna and J.M. Mallikarjuna / JAFM, Vol. 3, No. 2, pp. 23-32, 2010.

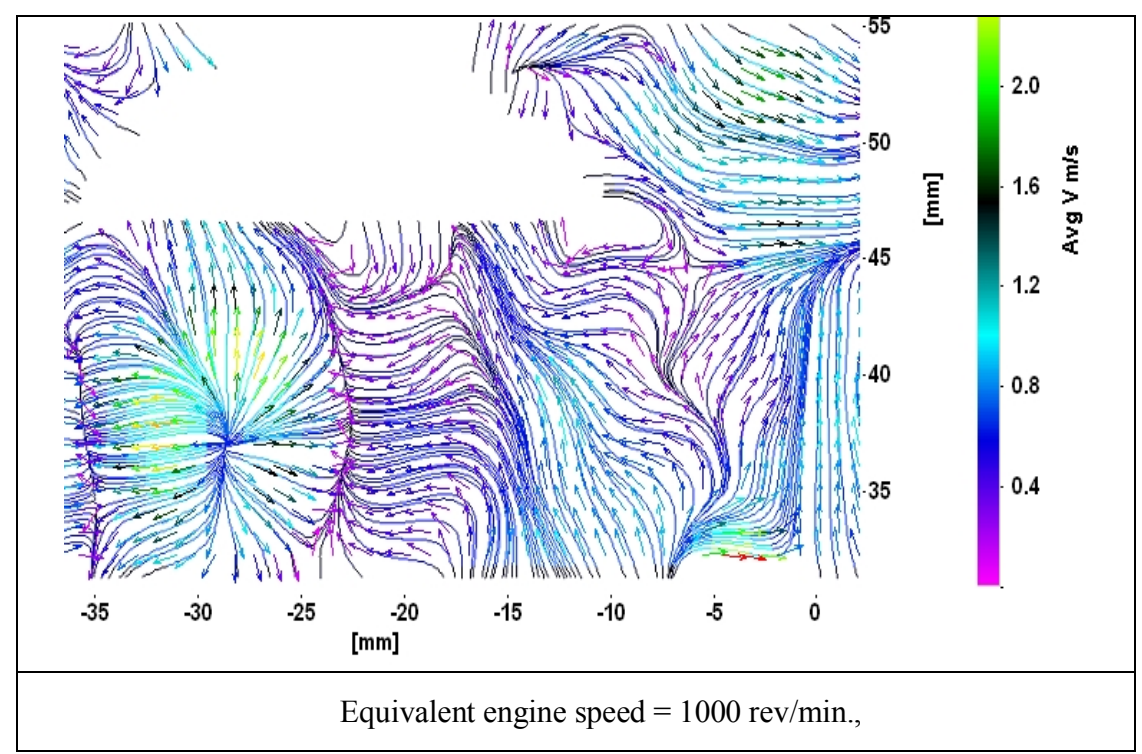

Fig. 5. Ensemble average velocity vector fields at $3 / 4^{\text {th }}$ intake valve opening

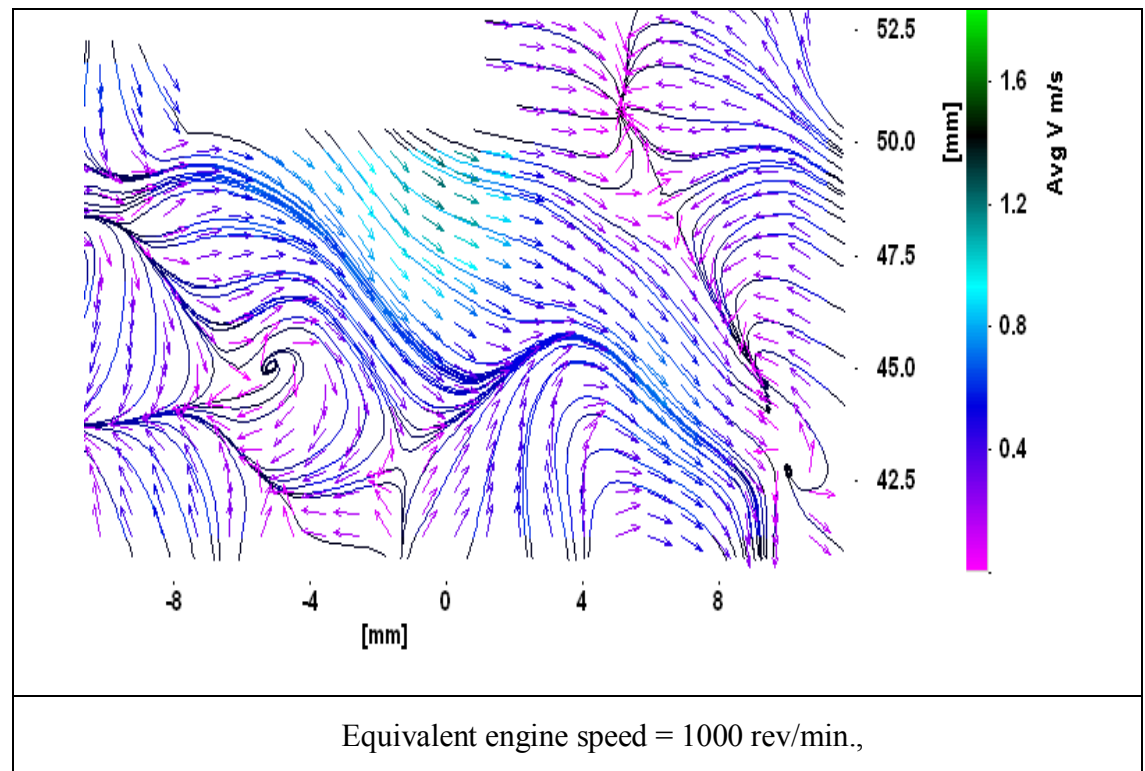

Fig. 6. Ensemble average velocity vector fields at half intake valve opening

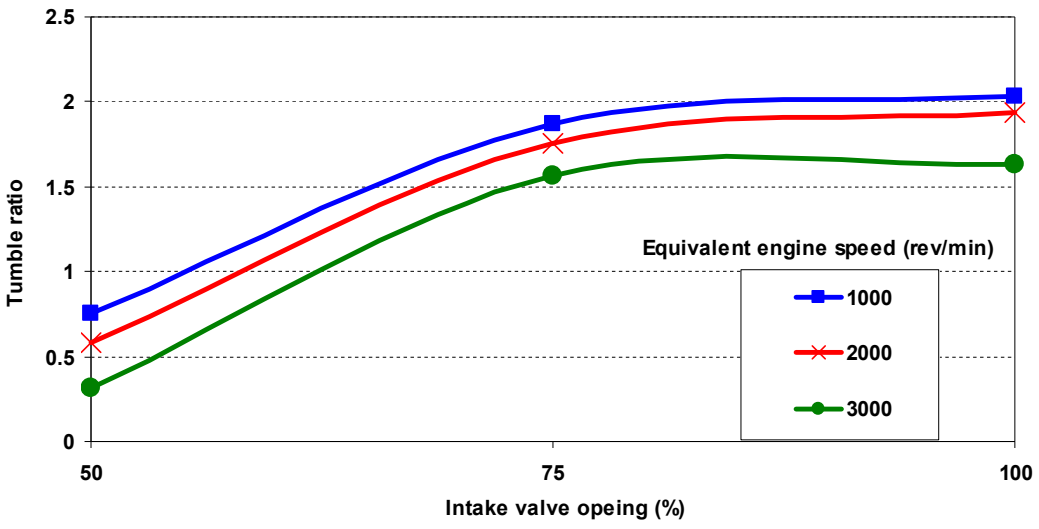

Fig. 7. Variation of tumble ratio with equivalent engine speeds and intake valve opening 
B.M. Krishna and J.M. Mallikarjuna / JAFM, Vol. 3, No. 2, pp. 23-32, 2010.

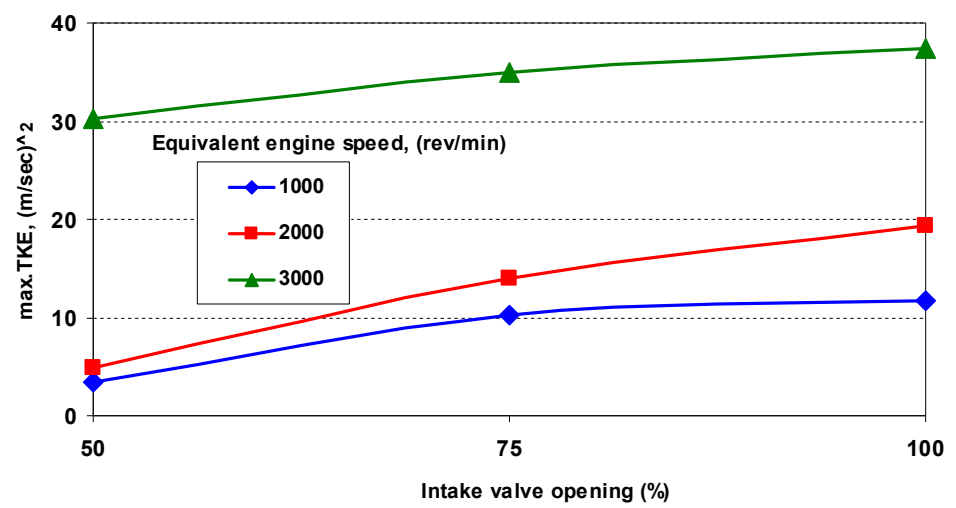

Fig. 8. Variation of maximum TKE with equivalent engine speeds and intake valve opening
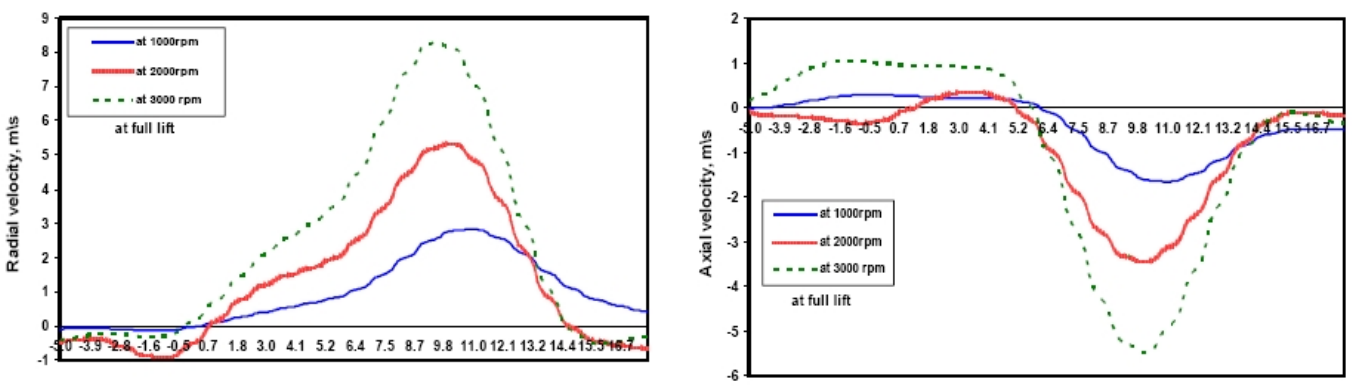

Horizontal location, $\mathrm{mm}$

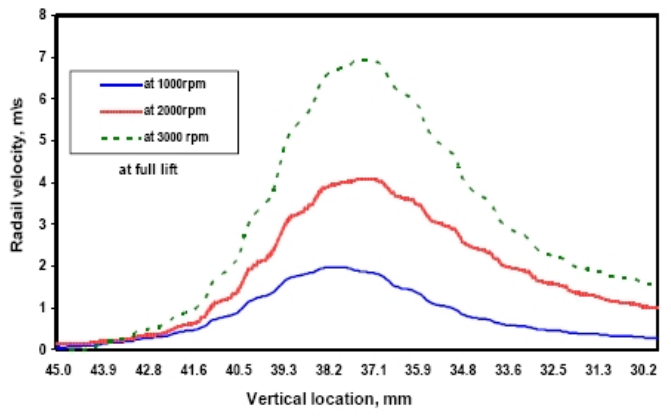

Horizontal location, $\mathrm{mm}$

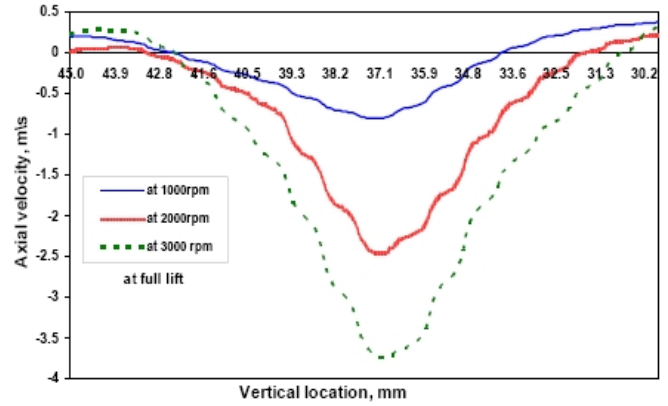

Fig. 9. Profiles of radial and axial velocities for various equivalent engine speeds at full intake valve opening 
B.M. Krishna and J.M. Mallikarjuna / JAFM, Vol. 3, No. 2, pp. 23-32, 2010.
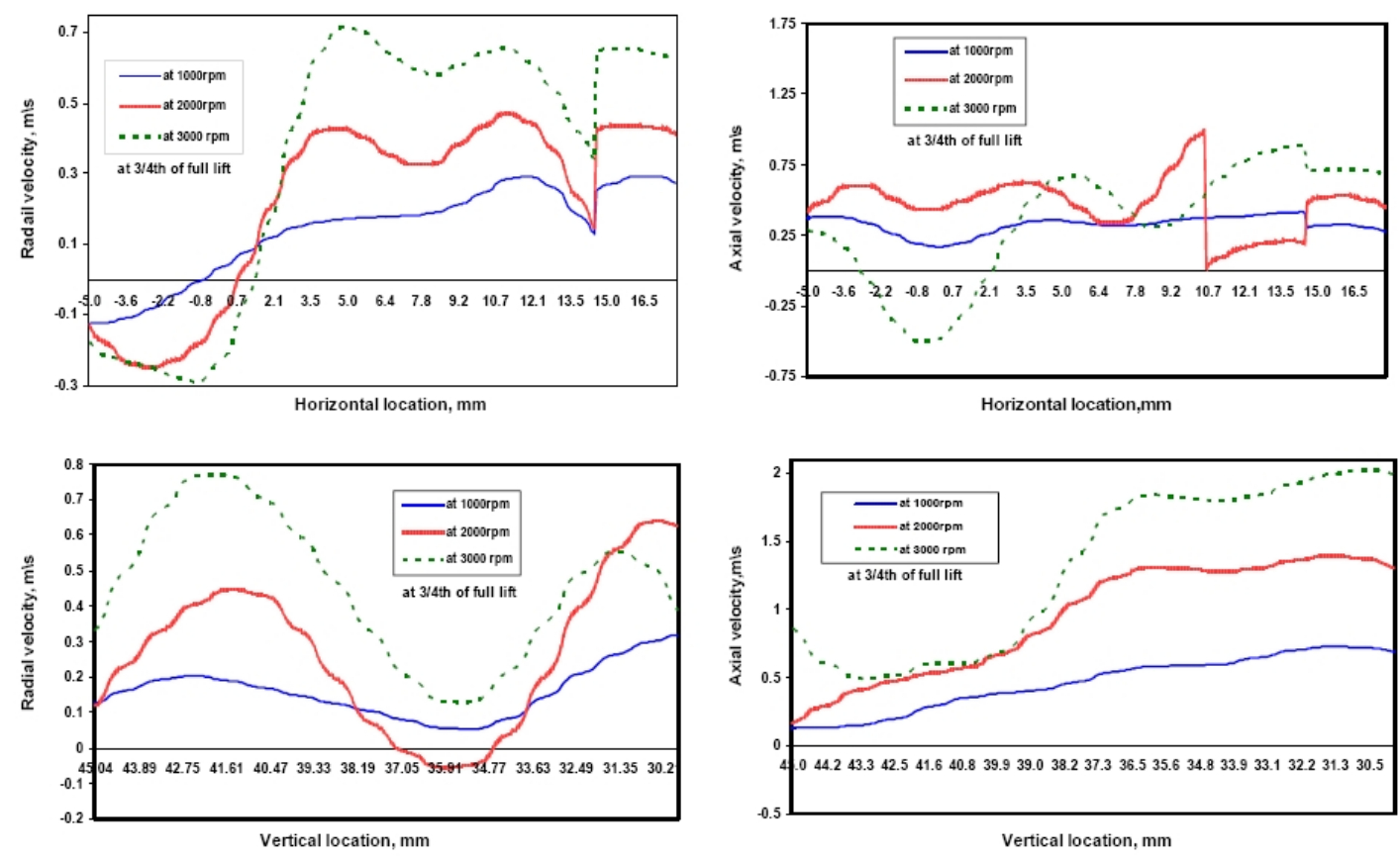

Fig. 10. Profiles of radial and axial velocities for various equivalent engine speeds at $3 / 4^{\text {th }}$ intake valve opening
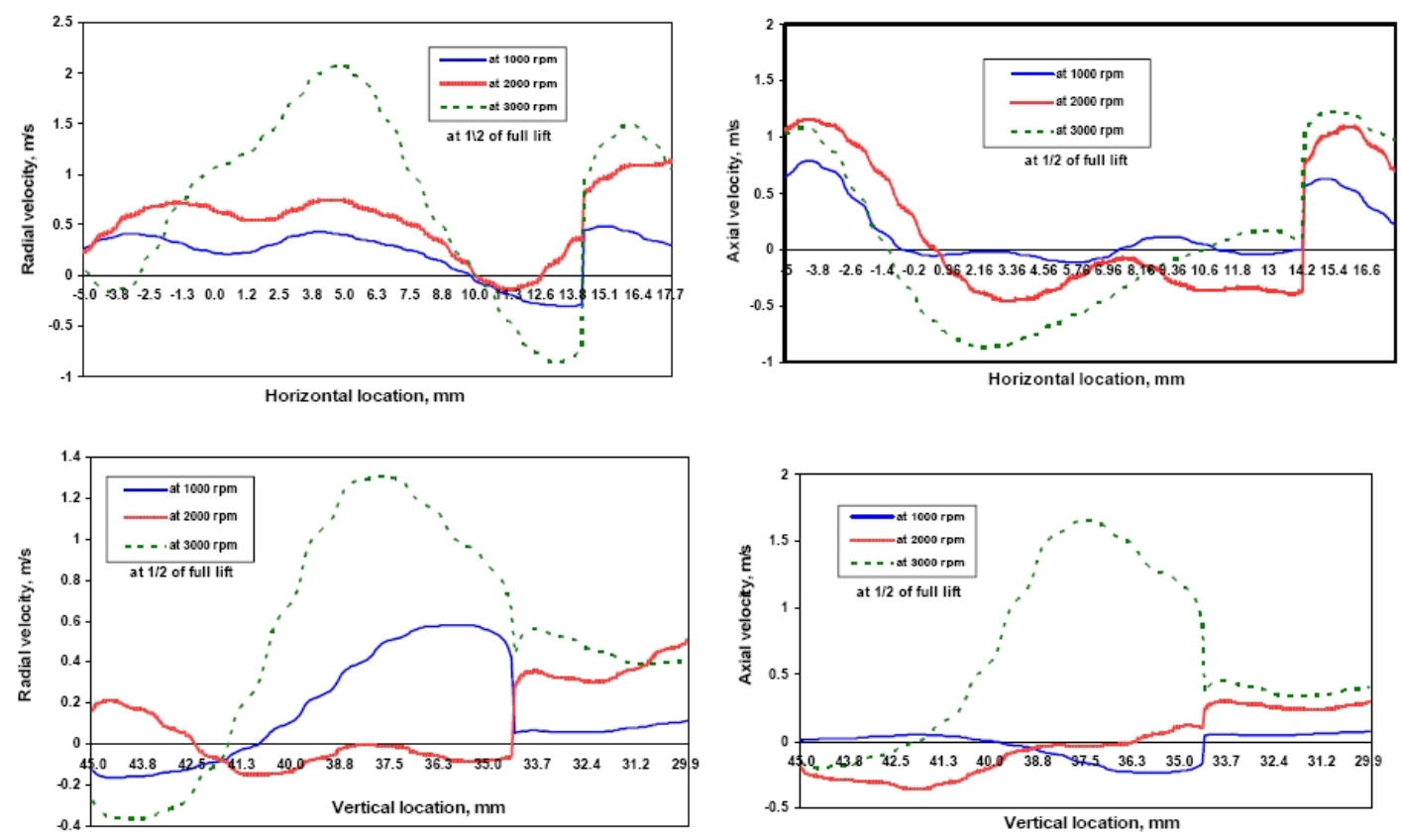

Fig. 11. Profiles of radial and axial velocities for various equivalent engine speeds at half intake valve opening 